\title{
DOCUMENTATION
}

\section{L'aménagement hydraulique de la vallée d'Aspe}

La Société des forces motrices de la Vallée d'Aspe a étudié un vaste programme d'ensemble pour l'aménagement de la Vallée du Gave d'Aspe dans les Basses-Pyrénées, qui s'étend sur une longueur de $50 \mathrm{~km}$. offrant une dénivellation totale de $1.490 \mathrm{~m}$. Ce projet comporte le fractionnement en 7 chutes successives, utilisces rlans les usines de :

Estaens $4000 \mathrm{CV}$, usine de haute chute à réservoir saisonnier Les Forges d'Abel ( $4.000 \mathrm{CV})$ et Baralet $(16.000 \mathrm{CV})$, usines a réserve journalière ; Eyguer Lesau (28.000 CV), Esquit $(6.000$ CV), Lourdios (10.000 CV); Escat (16.000 CV) et Asasp (1.600 CV), usine au fil de l'eau.

Ces usines sont énumérées dans leur ordre géographique, en descendant vers l'aval; celles d'Esquit, des Forges d'Abel et d'Estaens sont ell service, celle de Baralet sera mise en route en 1926, les autres sont en projet et l'on envisage, en outre, en amont l'Estaens une usine dont la chute sera la plus haute de France et d'autre part une centrale thermique de réserve.

L'énergie produite par ces usines, reliées entre elles par des lignes à $60.000 \mathrm{v}$, sera utilisée par les diverses sociétés de distribution de la région (Energie électrique dans les Basses-Pyrénées et les Landes, Cie du Gaz d'Agen, Sté hydroélec. des B. P. à Bayonne, Sté Pyrénéenne, dans le Gers), et la consommation Iocale, est en outre transmise à grande distance par le réseau à $150.000 \mathrm{v}$. des chemins de fer du Midi, auxquels les lignes de la Sté sont raccordées au poste élévateur de Hourat-Larens.

L'article, après étude géographique et hydrologique de l'ensemble du réseau projeté et de la partie actuellement exécutée aborde la description détaillée de l'usine d'Esquit et de ses aménagements hydrauliques. La chute brute utilisée est de $42 \mathrm{~m}$. avec débit moyen de $13,5 \mathrm{~m}^{3} / \mathrm{s}$. prélevé, par 2 prises distinctes, sur le gave d'Aspe et le gave de Lescun. Ce second cours d'eau cst traversé par le canal venant du premier par un siphon au delà duquel est situé l'ouvrage de raccordement. Le canal d'amenée mesure $2.500 \mathrm{~m}$. dont $1.300 \mathrm{~m}$. en souterrain, tout le parcours à ciel ouvert étant recouvert en outre d'une voûte de protection. Ce canal franchit le ruisseau d'Anitel par un pont-canal et aboutit tout près de l'usine, à la chambre de mise en charge, de $1.000 \mathrm{~m}^{3}$ de capacité, d'où part la conduite forcée, en tôle de $2 \mathrm{~m}$. de diamètre et de $70 \mathrm{~m}$. environ de longueur, pourvue à son orifice d'un reniflard. Les ouvrages sont pourvus des vannes de mancuvres, de charges, vannes de fond, etc.

L'usine génératrice, trés étudiée, au point de vue décoratif pour ne pas nuire au cachet de la vallée, renferme 3 groupes.

Les turbines de $2000 \mathrm{CV}$ à $500 \mathrm{t}$./m. sont du type Francis horizontal avec zone en partie à faire, elles ont été construites par "Constructions électriques de France".

Les alternateurs à $6.000 \mathrm{v}$. ainsi que tout le matériel électrique et l'appareillage ont été fournis par la Cle Electro-Mécanique.

Ces machines et les essais qu'elles ont subis sont décrits en détail, et de nombreuses vues de l'installation ainsi que le schéma complet accompagne l'article.

A une extrémité de la salle se trouvent les cellules du tableau à $6.000 \mathrm{~V}$., surmontées d'une estrade portant le pupitre de manœuvre qui se trouve ainsi placé directement au-dessus des interrupteurs commandés par chaîne et cable, suivant le type ordinaire de la C. M. E. 3 cellules correspondent aux alternateurs, une au couplage de 2 jeux de barre, 2 autres renferment l'appareillage de départ vers les transformateurs élévateurs en 1 celui d'un départ souterrain vers l'usine métallurgique de Bedons.

L'élévation de la tension de 6.000 à 66.000 volts est assuré par un groupe de 4 transformateurs monophasés, l'un formant réserve et pouvant être immédiatement connecté, par un jeu de sectionneurs sur l'une quelconque des 3 phases, en place d'un appareil avarié.

La puissance de ces transformateurs $6.000 / 38.000$ v. est de $1.700 \mathrm{KVA}$ chacun; l'article les décrit avec les essais subis et les résultats obtenus. Ils sont installés en cellules derrière le tableau à 6.000 v. au-dessus d'eux et placé, en cellules également, un interrupteur tripolaire à 3 bacs séparés pour le départ de la ligne aérienne à $66.000 \mathrm{v}$. Il est commandé électriquement du pupitre de manceuvre.

Aux cellules à $6.000 \mathrm{v}$. des alternateurs correspondent des régulateurs de tension Brown-Boveri et des régulateurs limiteurs de courant, de la même société, en vủe de protéger efficacement les machines contré les courts-circuits qui sont appelés à devenir très redoutables étant donné la puissance formidable des centrales réunies avec lesquelles ces machines travailleront en parallèle.

Revue des Revues.

G. G.

\section{Importance de l'accroissement de la charge des centrales électriques par suite du développement du chauffage électrique industriel}

Il y a 10 ans encore que la charge provenant du chauffage industriel était pratiquement négligeable aux Etats-Unis. Aujourd'hui cette charge figure au budget de chaque centrale et dans nombre de districts industriels c'est après les moteurs la charge la plus importante, et cette charge croit beaucoup plus rapidement que toute autre. I, 'auteur cite des chiffres en se basant principalement sur les résultats d'exploitation de la "Connecticut Light et Power Co " faisant ressortir depuis 1918 l'accroissement rapide de cette charge. La plus grande partie de cette charge étant représentée par des fours de fusion pour le laiton, puis par les fours pour traitements thermiques. De nombreux fours de $2.100 \mathrm{KW}$ sont actuellement en cours d'installation.

L'auteur fait ressortii les raisons légitimant cet accroissement : $1^{\circ}$ moins d'oxydation du métal ; $2^{\circ}$ moindre consommation d'acide au décapage; $3^{\circ}$ moins de temps pour l'opération du décapage $4^{\circ}$ économie de main-d'œuvre; $5^{\circ}$ réduction des pertes de métal : $6^{\circ}$ améliorations des conditions de travail ; $7^{\circ}$ produits plus homogènes, etc.

Si l'accroissement de la charge due au chauffage électrique continue à progresser suivant le degré de ces dernières années, on peut estimer qu'aux Etats-Unis on aura dès 1931 une charge "Chauffage " égale à la charge "Moteurs".

Aux avantages ci-dessus énumérés s'ajoutent encore des avantages d'ordre économique et de production comme il est indiqué par des exemples.

R. M. K.

Electrical World, 23 octôbre 1926.

\section{La nouvelle grande centrale thermo-électrique de Rummeisburg}

L'accroissement de la consommation en courant qui se manifesta à Berlin au lendemain de la période d'inflation (1923) obligea la municipalité de la ville en 1925 de décider la construction d'une nouvelles grande centrale actuellement en cours d'exécution à Rummelsburg, dans la banlieue de la ville. Une première tranche du programme comporte pour 1926-1927 l'installation de trois groupes turbo-alternateurs d'une puissance unitaire de 100.000 KVA, soit de $240.000 \mathrm{KW}$ pour un cos de 0,8 . Une surcharge est admissible pour les turbines permettant aux heures de pointe d'éclairage de donner $270.000 \mathrm{KW}$ pour un cos de 0,9 . 
Le développement ultérieur de la centrale prévoit l'installation de six groupes semblables. Les perfectionnements les plus récents de la technique américaine aussi bien qu'européenne seront évidemment incorporés dans la conception et linstallation de cette centrale.

6 pages de texte et 9 clichés exposent clairement toutes les paricularites de cette importante centrale : emplacement, manu- tention du charbon, chaudières, turbines, etc... sont successivement examinés.

I.es groupes turbo-alternateurs sont du type a triple expansion, un alternateur est accouplé sur l'arbre commun à la turbine hatut pression et a l'arbre de la turbine moyenne pression, tandis que le deuxième alternateur de même puissance que le premier est accouplé sur l'arbre commun aux deux turbines basse pression.

\section{Calcul des fondations des pylônes des lignes de transmission d'énergie électrique}

Ces articles contiennent l'exposé d'une étude très détaillée dé ce problème, pour lequel l'auteur estime qu'il serait utile do poursuivre une série d'essais, comme d'ailleurs cela a été commencé en Allemagne et en Suisse dans des circonstances qu'il relate afin déviter un gaspillage de matériaux qui n'augmente pas toujours la sécurité de la construction, dù à l'incertitude des méthodes généralement utilisées aujourd'hui.

Il commence par aborder théoriquement l'étude de l'action des verres, poussée et butée, en établissant à ce sujet une série de théories fondamentales.

I.e calcul des fondations des pylônes à pied cintré peut se faire en partant de 2 considérations différentes :

A. - Support considéré comme reposant sur le sol, où l'on ne peut compter que les poids du pylone et de ses agrès comme forces concourant à la stabilité.

B. - Support considéré comme encastré dans le sol, glonnant lieu à 2 groupes distincts de méthodes de calcul.

Les méthodes du $1^{\text {er }}$ groupe ont pour base les travaux d'Engel et de Mohr relatifs à une verge enfoncée dans le sol. Ils ont donné lieu à une série d'essais effectués en 1913 par l'administration allemande sous la direction du $\mathrm{D}^{\mathrm{r}}$ Frahlich. 2 méthodes d'essais en découlent, l'une appliquée en Allemagne, l'autre en Fránce, différentes par la formule fondamentale et les valeurs des coefficients faisant intervenir la nature des terrains.

Les méthodes du $2^{2}$ groupe ont pour base les études théoriques d'Andrée. Elles ont été considérablement développées et mises au point par une série d'essais elfectués en Suisse, à Goësgen, qui ont conduit la commission suisse pour la révision des prescriptions fédérales concernant les installations à courant fort à préciser des formules et un tableau de coefficient, formules à portées desquelles fut tracé une abaque permettant le calcul très rapide des fondations dans les divers cas qui peuvent se présenter.

A la suite de cette étude, l'auteur passe à celle du calcul des fondations de pylones à pieds multiples, et de ses différents cas, sur lesquels ont porté également les essais de Gosgen.

L'article se termine par une longue énumération bibliographique des ouvrages et articles traitant de ces matières et qui ont été consultés par la mise au point de son importante étude.

R. G. E., 6 et 13 novembre 1926 .

H. C.

\section{L'usine génératrice de Chancy-Pougny, description des alternateurs et résultats de leurs essai $i_{s}$}

Les cinc alternateurs, à axe vertical, de $7.000 \mathrm{KVA}$ installés pans cette usine ont été construits par les établissements Schneider. Leur tension est de 10.000-11.000 volts et se trouvent à $83,3 \mathrm{Hm}$. (hauteur de chute $8 \mathrm{~m} .87$ ), ce qui, pour $50 \mathrm{p}$. s. donne 72 pôles au rotor. Le poids total, y compris l'excitatrice est de 220 t. dont 90 pour la partie tournante. Le piyot supérieur, sur les croisillons, porte, outre ce poids, celui du rotor de la turbine, soit 40 t. et la poussée dynamique de l'eau, soit environ $100 \mathrm{t}$.

L'auteur décrit en détail la carcasse (en 4 pièces) avec les dispositions des tôles actives et de la ventilation, la roue polaire en 4 parties assemblées dont le P. $D^{2}$ atteint $2.000 .000 \mathrm{~kg} / \mathrm{m}^{2}$, les bobinages et le freinage à air comprimé permettant d'immobiliser la partie tournante. Des dessins de la machine accompagnent la description. La vitesse d'emballement prévue est de $166 \mathrm{Hm}$.

Puis la description des essais est présentée en détails, avec les différentes caractéristiques qui sont reproduites, en même temps que sont signalés le mode opératoire utilisé pour les relever et les particularités rencontrées :

Caractéristiques à vide de l'excitatrice, avec coude vers la partie inférieure, nécessaire, avec l'excitation shunt, pour assurer un fonctionnement stable aux faibles valeurs du courant d'exci tation, ce qui est indispensable pour la mise sous tension de la longue ligne à 120.000 volts avec un seul alternateur. On a été amené en outre à augmenter un peu l'entrefer de l'excitatrice en vue de l'adaptation à un régulateur de tension déterminé.

\section{La turbine à impulsion et réaction}

Cette machine est particulièrement digne de remarque par le fait que sa vitesse de rotation est égale à $3.000 \mathrm{t}$. : $\mathrm{mn}$, ce qui constitue un record. A des puissances comprises entre 12.000 et $20.000 \mathrm{KW}$, la consommation de vapeur par kilowatt-heure varie de 5 à $5,2 \mathrm{~kg}$; le rendement thermique atteint 28 pour 100 environ. La machine est du type à deux corps : la partie à haute pression développe 60 pour 100 de la puissance, elle est du type à impulsion; la partie à basse pression, par contre, est du type à répulsion, et est alimentée au centre, la vapeur s'écoulant vers les deux extrémités. Le rotor à haute pression est d'une seule pièce; les diverses roues sont prises dans la masse, ce qui a permis d'épargner la place en longueur tout en ménageant 12 étages d'expansion. Cette construction a encore l'avantage d'augmenter la rigidité du rotor et de diminuer les fuites de vapeur d'un étage à l'autre. Les aubes directrices sont en acier doux, l'angle de sortie étant de 14 dégrés;
Caractéristiques de l'alternateur. Le stator est ell étoile avec neutre sortie en vue de l'application éventuelle de la protection différentielle Métry Price et de la mise à la terre du neutre.

L'auteur s'étend sur la prédétermination du courant de courtcircuit instantané et de la chute de tension, valeurs sur lesquelles des conditions précises étaient imposées pour le marché.

La prédétermination de la chute de tension a été faite d'abord par la méthode de Pottier (appliquée suivant les indications de M. Fallou, puis par celle de Rothert). On a constaté, une fois de plus, que la méthode de Pottier donne des résultats sensiblement plus exacts que la seconde.

La mesure des rendements enfin était d'importance capitale, car il fallait exactement parer l'alternateur, pour parvenir par la suite à déterminer le rendement de la turbine hydraulique, d'autre part, le tirage de grosses unités présente une assez grande difficulté du fait de l'incertitude sur l'élévation des pertes supplémentaires. Cette question a été étudiée par M. Roth, et une méthode de correction a été proposée.

Les pertes dans le cuivre ont été déterminées par mesure des résistances. Les pertes dans le fer et les pertes mécaniques l'ont été de 2 façons : d'abord, en entraînant l'alternateur fonctionnant en moteur synchrone par l'alternateur d'un groupe voisin, méthode qui n'est pas sans présenter, au démarrage, des dangers pour le pivot; en second lieu par la méthode de ralentissement.

\section{R. G. E., 30 octobre 1926.}

B.

\section{de 20.000 kilowatts de Bradford}

les aubes mobiles sont en acier au nickel; leur vitesse maximum ne dépasse pas $170 \mathrm{~m}$. : s, à pleine charge; la vapeur est détendue dans la turbine à haute pression jusqu'à une valeur voisine de la pression atmosphérique. Le rotor de la partie à basse pression est également pris dans la masse; la vitesse, maximum des aubes atteint $260 \mathrm{~m}$.: s. Les paliers sont munis de coussinets en fonte garnie de métal blanc; la surface portante est très courte et ils peuvent être considérés comme constituant des coussinets à rotule. La poussée axiale est équilibrée par un palier de butée du type Michell qui peut être déplacé longitudinalement d'une petite quantité pour le réglage des rotors dans les stators des turbines. Une planche et de nombreuses figures dans la texte completent la description.

Engineering, 15 octobre 1926, t. cxxrr, p. 465-469, 3.800 mots, 30 fig. 


\section{Moteurs synchrones à très grand couple de démarrage}

Les quatre cas à envisager lorsqu'il s'agit de démarrer avec des moteurs à courant alternatif devant exercer un couple supérieur à $60 \%$ du couple normal sont : $1^{\circ}$ l'emploi d'un moteur d'inducLion; $2^{\circ}$ l'emploi d'un moteur synchrone ordinaire avec accouplement magnétique; $3^{\circ}$ l'emploi d'un moteur synchrone à double cage; $4^{0}$ l'emploi d'un moteur synchrone à stator freiné. C'est cette solution que l'auteur envisage particulièrement en la comparant avec chacune des trois autres. Le principe d'un moteur synchrone à stator freiné est d'avoir un stator conçu de façon à pouvoir tourner à la vitesse synchrone, le rotor étant à cage d'écureuil et le stator prévu pour pouvoir être freinć progressivement jusqu'à blocage complet. Les phases du démarrage sont : $1^{\circ}$ démarrage du stator à tension réduite; $2^{\circ}$ accrochage; $3^{\circ}$ passage à plaine tension (le rotor est alors fixe et le stator tourne à la vitesse de synchronisme) et réglage de l'excitation (la vitesse relative statorrotor demeure la même mais celle du stator commence par freinage à diminuer pendant que le rotor prend une vitesse croissante): $4^{\circ}$ freinage. Les avantages particuliers au système sont exposés ainsi que les indications sur les prix et sur quelques-unes des principales applications.

A. P.

\section{Note sur la comparaison des rendements des turbines à vapeur}

I'auteur montre que, si l'on suppose le rendement d'aubage constant tout le long a'une turbine, sa détermination peut être rapicle et facile. Le rendement thermique qu'il envisage est le rapport de la puissance sur l'arbre à l'énergie théoriquement disponible dans la vapeur entre son entrée dans la turbine et sa sortie de l'aubage. Le facteur de réchauffage interne est le rapport de la somme des chutes de chaleur dans chaque rangée d'aubes, à la chute le chaleur totale. Il est toujours plus grand que l'unité, et dépend du rendement de l'aubage. L'emploi des hautes températures d'admission, c'est-à-dire des surchauffes élevées, entraîne l'avantage d'une diminution de l'humidité pendant la détente, tandis que les hautes pressions ont l'inconvénient d'accroître les fuites. L'ar- ticle est accompagné de graphiques donnant : $1^{\circ}$ la variation de la chaleur totale et de l'entropie en fonction de la pression d'admission pour divers rendements d'aubage; $2^{\circ}$ la relation entre le rendement d'aubage, le facteur de réchauffage interne et la température d'admission, pour diverses températures d'admission: $3^{\circ}$ les rendements thermique et d'aubage dans le cycle Rankine et le cycle à extraction, pour diverses conditions de fonctionnement. Ces divers graphiques permettent de traiter d'une façon rapide les divers problèmes de comparaison.

Ernest-L. Robinson, G. E. R:, juillet 1926, t. xxix, p. 503-510, 3.500 mots, 10 fig.

\section{Les accumulateurs de chaleur}

Dans cette communication, l'auteur examine principalement l'emploi des accumulateurs de chaleur. Il étudie d'abord le cas de l'accumulation à courte durée (alimentation d'un chauffage par une source intermittente de chaleur) et montre la méthode de calcul de l'installation suivant la durée des interruptions. L'accumulateur Rateau rentre dans cette catégorie d'appareils. L'auteur étudie ensuite l'appareil Ruths permettant l'accumulation pendant des périodes de plusieurs heures. Il décrit deux exemples d'installation de cet appareil. Il passe alors aux accumulateurs à eau chaude et, après avoir décrit le principe d'un de ces appareils, il indique plusieurs applications possibles. Il examine enfin l'application des accumulateurs à une usine génératrice électrique combinée avec une distribution de chauffage urbain.

A. Beaurienne. Bulletin de la Société des Ingénieurs civils de France, juillet-août, t. Lxxix, p. 491-518, 10.000 mots, 15 fig.

\section{Transformateurs de 28.866 kilovolts-ampères, à 220.000 volts, pour la Pensylvania Power and Light Company}

Ce transformateur est monophasé et fait partie d'un groupe dont la puissance apparente totale est 86.600 KVA. L'ensemble peut fournir plusieurs tensions. Dans ce but, le transformateur décrit possède trois enroulements correspondant aux tensions de 11,66 et $220 \mathrm{KV}$. La protection contre les ondes isolées de tension a été spécialement étudiée et a abouti à l'emploi d'écrans électro- statiques, placés sur les enroulements dans le voisinage des bornes. L'isolement aux extrémités des bobinages est obtenu à l'aide de bakélite comprimée; les enroulements à 11 et $66 \mathrm{KV}$ sont munis d'indicateurs de température.

F.-F. Brand, G. E. R., juillet 1926 , t. xxıx, p. 499-502, 2.300 mots $5 \mathrm{fig}$

\section{Deux transformateurs de 60.000 kilovolts-ampères, munis d'un réglage de la puissance, pour la Philadelphia Electric Company}

Ces transformateurs, avec un dispositif de changement du rapport de transformation en charge, sont installés sur une ligne de 66.000 v. Chacun se compose de trois éléments à courant monophasé de $20.000 \mathrm{KVA}$, fonctionnant entre $13.800 \mathrm{v}$. et $72.450 \mathrm{v}$. Les primaires sont reliés en triangle et les secondaires, en étoile. Le système de réglage de la tension constitue la caractéristique la plus intéressante de l'installation. Les primaires de chaque phase alimentent une impédance fractionnée en 9 sections. Deux contacts peuvent se déplacer et embrasser entre eux un nombre variable de ces sections. Ils alimentent, d'autre part, le primaire d'un transformateur dont le secondaire est en série avec celui du transformateur principal. Le déplacement des contacts permet d'alimenter le transformateur additionnel à l'aide d'une tension variable et, par suite, d'ajouter une tension variable à la tension principale. Une liaison mécanique assure le synchronisme des opérations sur les trois phases, nécessaire pour éviter les déséquilibrages.

Arthur Palme et H.-O. Stephens. G. E. R., septembre 1926 , t. $\mathrm{xxIX}$, p. 634-638, 2.400 mots, 6 fig.

\section{Détecteur à gaz pour lampes électriques de sécurité des mineurs}

Le dispositif, inventé et mis au point par M. Gulliford comporte un récipient perforé dans lequel se trouve un fusible entouré par un cylindre en treillis. Ce fusible, au moyen d'un interrupteur, peut être mis en série avec le filament de la lampe aussi souvent quie nécessaire, et, si l'atmosphère ne se révèle pas grisouteux, lorsque le fusible est dans le circuit, la lampe donne moins de lumiere que lorsqu'elle est connectée directement aux bornes de la batterie, et le fusible est rouge sombre. S'il y a traces légères de grisou, le fusible devient plus chaud et son éclat plus intense, mais celui de la lampe diminue. Enfin, si la proportion de grisou dépasse $2,5 \%$ la lampe s'éteint, attirant immédiatement l'attention des mineurs. Le fusible étant alors court-circuité, les mineurs peuvent quitter sans encombre la zone dangereuse et donner l'alarme.

Engineering, 12 mars 1926.
J. B. 


\section{Les usines hydrauliques de Sao-Paulo}

Dans le but de remédier à sa pénurie d’énergie, la "Sao Paulo light and Power Co "exécute actuellement d'importants travaux d'aménagements hydrauliques comportant une centrale à Cubatao, au pied de la Sierra, équipée pour utiliser une chute du Rio Pedras constituée par des eaux en provenance des rivieres Padra et Granelle - reliées au réservoir de Santo Amaro - et de divers petits torrents sur lesquels sont prévus des barrages d'importance varia ble. L'ensemble des travaux doit être exéculé en 3 étapes en commençant par l'exécution du barrage de Rio Pedras, et en ter minant par celle de Santo Amaro, sur le Rio Grande.

La centrale doit comporter, en 1 étape, 2 unités de $40.000 \mathrm{CV}$

The Engineer, 8 décembre 1926.

\section{Les noyaux en ferro-nickel " hypernik )}

Généralement interposés entre les appareils de mesure et les câbles $H$. T. destinés à des mesures d'intensité, ces transfos sont munis de noyaux en acier siliceux, assez faiblement perméables. et qui comportent, à forte charge, des inductions trop élevées, d'où découlent des courants magnétisants avec, dans le fer, des pertes très élevées - ce qui accroit, dans de fortes proportions. les erreurs de déphasage - alors qu'à faible charge, avec une induction également faible, et les changements correspondants dans les conditions de fonctionnement. leur sensibilité est com-

\section{dans les transformateurs de fréquence}

\section{plètement modifiée.}

Dans son intéressant article, $M$. Th. Spooner expose que l'hypernik (nouvel alliage nickel et fer) permet d'obtenir des noyau. à perméabilité élevée pour une faible induction. ramenant à $30 \%$ de ce qu'elles sont, avec les noyaux siliceux, les erreurs de dépliásage précitées, et avec lesquelles le rapport minimum pour lequel on ait observé un fonctionnement normal de l'appareil est de $200 / 5$. Journ. of the Am. Institute of Electr. Engineers, juin 1926.

J. 13.

\section{Les compensateurs synchrones}

Dans cette sous-station de l'Union d'Electricité (qui almente toute la région parisienne - sauf Paris -) fonctionnent depuis peu 2 compensateurs synchrones de $7.000 \mathrm{KVA}$. ayant pour objet la régularisation de la tension en ce point de réseau.

Après avoir exposé comment un compensateur semblable possédant une excitation dûment réglée - peut maintenir constante la tension d'extrémité d'une ligne sujette à des variations de tension (en faisant varier le déphasage par rapport à la ten-

\section{de 7.000 KVA de Billancourt}

sion), M. Doutrier donne la description des 2 compensateurs en question, qui reçoivent du triphasé $10.000 \mathrm{~V}$. 50 p. s. par l'intermédiaire de transfos abaisseurs $60.000 / 10.000$, et tournent à la vitesse de régime de $1.000 \mathrm{t}$. m., après démarrage au moyen de moteurs auxiliaires, asynchrones, montés en bout d'arbre. à l'opposé des excitatrices.

J. B.

R. G. E., 17 juillet 1926.

\section{Le matériel roulant des "Montreux-Oberland Bernois"}

Ce matériel est en progrès constants. On sait que le réseau est à voie métrique et comporte $76 \mathrm{~km}$. de lignes électrifiées, dont les rampes atteignent jusqu'à $70 \%$ sur d'assez longs parcours. Sur ces déclivités sont remorqués des trains de 250 T. dont le travail spécifique des moteurs par tonne kilométrique remorquée atteint 65 Wh. (non compris démarrages et services accessoires).

Dans cette étude, l'auteur expose les caractéristiques saillantes de nouvelles automotrices - en service depuis près de 2 ans. -
La tare de ces automotrices atteint 36 T. Les moteurs - alimentés sous $750 \mathrm{~V}$. - sont au nombre de 4 ( 2 sur chaque bogie). Ils fournissent, chacun, sur l'essieu-moteur, $110 \mathrm{CV}$. en régime permanent, et 145 en pointe unihoraire, puissance particulïèrement élevée si l'on tient compte du peu d'emplacement disponible sur des bogies de voie de $1 \mathrm{~m}$.

Industrie Electrique, 25 décembre 1926.

\section{Les forces hydrauliques de la Finlande}

Les disponibilités de la Finlande sont évaluées à environ $700.000 \mathrm{KW}$, dont à peine $7 \%$ sont utilisés. La presque totalité de cette puissance est réalisable dans le bassin du lac Ladaga; sur ses 3 affluents, en particulier, sur le Vnoski qui, avec un B. V. de $80.000 \mathrm{~km}^{2}$ présente un débit de $460 \div 640 \mathrm{~m}^{3} / \mathrm{sec}$, régularisable par l'aménagement du lac Sä̈ma (superficie : $7.000 \mathrm{~km}^{2}$ ). Sur cette rivière seulement sont prévues 4 centrales utilisant une chute totale de $62 \mathrm{~m}$. 50 sur un parcours de $26 \mathrm{~km}$. Parmi ces usines, celle d'Imatra - utilisant la plus haute chute $(24 \mathrm{~m}$.) est déjà construite et contient 3 groupes générateurs de chacun $24.000 \mathrm{KVA}$. à $11.000 \mathrm{~V} .-170 \mathrm{t}$. m. - $(\cos \varphi=0,8)$. L'énergie produite est transportée à $120.000 \mathrm{~V}$., au moyen d'une ligne dont les 3 conducteurs sont disposés en une nappe horizontale de manière à éviter tout contact entre les phases pouvant provenir de charges de glace excessives.

Elettrotecnica, 25 avril 1926.

\section{Les grosses turbines à hélice de la centrale de Kachlet}

La centrale de Kachlet, près de Passau, sur le Danube, exploite une chute de $7 \mathrm{~m} .85$ sur le Danube et est appelée à comporter, après équipement complet, 8 groupes générateurs de $6.000 \mathrm{KW}$. $-6.300 \mathrm{~V} .-(\cos \varphi-0,7)$ constitués par des turbines à hélice utilisant chacune un débit de $87,5 \mathrm{~m}^{3} / \mathrm{sec}$. en régime normal et pouvant utiliser juscqu'à $92,4 \mathrm{~m}^{3}$. sous $9,20 \mathrm{~m}$. de chute en hautes eaux moyennes; ceci à la vitesse normale de 75 tours. La roue présente un diamètre de $4,60 \mathrm{~m}$. et pèse environ 22 tonnes ; l'arbre vertical, long de plus de $8 \mathrm{~m}$., a un diametre de $475 \mathrm{~m}$. Chaque turbine a un distributeur fixe à 6 aubes, pesant chacun $2 \mathrm{~T}$., et 24 aubes mobiles disposées entre les autres, hautes de 1,67 m. et commandées par servo-moteur à huile.

Elettrotecnica, 15 décembre 1926 (Cron).

\section{La nouvelle centrale thermique de Langerbrugge}

Cette usine est une des plus intéressantes d'Europe au point de vue de l'utilisation de la vapeur à très hautes pressions.

Les chaudières - de construction Babcok et Wilcox - sont du type marine, avec réchauffeurs d'air et fournissent de la vapeur à $442^{\circ} \mathrm{C}$. sous $51 \mathrm{kgs} / \mathrm{cm}^{2}$.

L'eau d'alimentation est chauffée à $200^{\circ} \mathrm{C}$. par la vapeur, avant son admission dans la chaudière - ce qui supprime l'économiseur.
- Les 3 corps de chaudière ont $105 \mathrm{~m}$. de cliamètre et sont renforcés par une plaque tubulaire de $45 \mathrm{~m} / \mathrm{m}$ d'épaisseur. Les tubes ont $76 \mathrm{~m} / \mathrm{m}$ de diamètre.

Les chaudières - comme la turbine (de construction Brown Boveri) - - sont en service commercial depuis plusieurs mois, à l'entière satisfaction des exploitants.

Engineer, 4 juin 1926. 


\section{Conservation des fourrages par voie électrique}

Le procédé vise à empêcher la formation d'acide butyrique et la production correspondante de bactéries. Pour cela, le fourrage doit etre fortcment comprimé dans un silo dans lequel on fait passer, par la masse végétale, un courant alternatif de $250 / 500$ volts pendant 24 à 48 heures au moyen d'une électrode à extrémités métallicques. Le transfo d'alimentation est prévu pour une connexion série-parallele afin d'obtenir les 2 tensions. La consom- mation atteint 3 à $6 \mathrm{KWH}$. par quintal de fourrage traité.

Les essais tentés jusqu'à ce jour en Suisse ont été pleinement satisfaisants (sauf dans le cas où le lait des vaches est utilisé pour la fabrication du gruyère); mais le prix de revient, élevé, appelle néanmoins de sérieuses améliorations. J. B.

Bulletin Association Suisse des Electriciens, décembre 1925.

\section{Calcul des courants de court-circuit sur les réseaux triphasés}

Supposant les courants de court-circuit symétriques et conduisant à la notion (plutôt critiquable) de puissance de coupure exprimée en KVA, les méthodes généralement employées pour ces calculs fournissent presque toujours cles résultats faux. Après l'avoir rappelé et démontré, l'auteur de cette intéressante étude développe la méthode nouvelle des "coordonnés symétriques " et son application à ces calculs des courants de courts circuits rlans les réseaux triphasés, tant entre 2 phases qu'entre phase terre ; puis il donne, à titre d'exemple d'application de la méthode, la solution des divers problèmes de dimensionnement des bobines ou des résistances de point neutre.

Bulletin-Société Française des Electriciens, septembre 1926.

\section{Progrès des condensateurs statiques pour l'amélioration des facteurs individuels de puissance des moteurs}

L'étude de M. Marbury, après un exposé général de la question et des diverses tendances que comporte sa solution, s'étend particulièrement sur les progrès récents que lui a fait faire la "Westinghouse Electrid and Mfg $C^{\circ}$ "par l'application des condenseurs correcteurs du cos o.

Ses condensateurs de protection sont établis par petites unités. pouvant fonctionner solidairement mais pour chacune desquelles a été appliqué un procédé de fabrication spécial mettant l'ensemble à l'abri d'un accident survenu à une unité. La capacité ainsi réalisée comporte, pour chaque section, une protection par fusibles de même que des sectionneurs classiques permettant la localisation et l'élimination de l'unité détériorée sous le simple soupçon d'une défectuosité, ou à premier besoin d'un essai:

J. B.

Electr. Journal, mai 1926.

\section{L'accident, et la résistance, du}

On se rappelle sans doute la destruction, survenue l'année (lernière, - et attribuée au gel, - du barrage de Gem Lake, du type à A. M., et dont la construction remontait à 1916 seulement. Après d'infructueux travaux de réparations, on dut le transformer en barrage à gravité, car, situé à $2.750 \mathrm{~m}$. d'altitude, il supporte, en hiver, des températures négatives descendant jusqu'à $-30^{\circ}$, pendant de longues périodes.

Une intéressante controverse s'est constituée, dans le $B$. T. de la

\section{barrage de Gem Lake (Cal.)}

Suisse Romande au sujet de cet accident, à la suite d'informations parues dans les Annali $L L$. PP. (juillet 1925) qui présentaient le barrage à A. M. comme trop mince; et dans cette controverse, nous voyons M. Sarrasin critiquer, au contraire, le trop de surbaissement de ses arches en mème temps que leur trop grande épaisseur, et $M$. Stucky prend également position sur la question, avec son autorité habituelle.

J. B.

B. T. de la Suisse Romande, 10 juin 1923, décembre 1926.

\section{Décharge brusque d'un groupe électrogène $=$ Pertubations de vitesse}

Ces perturbations sont fort importantes à connaitre, afin de pouvoir déterminer la vitesse maxima du groupe dans le cas d'une brusque décharge du réseau alimenté par le groupe.

La détermination de cette vitesse maximum fait l'objet de l'étude de M. Barbillion, qui, tout d'abord, établit l'équation générale définissant une perturbation puis indique une excellente détermination graphique de la vitesse qui correspond au maximum de rendement d'une turbine hydraulique pour une admission donnée, et donne, finalement, une étude fort approfondie de la décharge complète, à laquelle nous renverrons nos lecteurs.

Revue de l'Industrie Minérale, $1^{\text {er }}$ septembre 1926.

\section{Le freinage des tramways}

Intéressante comparaison des 2 modes de freinage classiques, de ces sortes de convois : freinage pneumatique, à l'A. c. et freinage électriq̣ue.

En ce qui concerne ce dernier, l'auteur expose les diverses manières de le réaliser (mise en circulation des moteurs - débitant sur des résistances - ; action d'un électro sur freins à patin ou sur disques de freinagc, etc...) mais souligne que, tous, impli-

\section{et des trains sur routes}

quent un appareillage et des moteurs entretenus en excellent état, - ce qui n'est pas toujours le cas dans les Compagnies secondaires : - alors que le freinage pneumatique, quoique plus coùteux comme installation et comme entretien, étant indépendant de cet appareillage autant que des moteurs, donne très généralement plus de sécurité que l'autre.

J. B.'

Schw. Bzg., 14 aoùt 1926.

\section{L'usine hydroélectrique d'Eguzon et l'électrification du P. $O$.}

Dans cette remarquable étude, l'auteur passe en revue les différents travaux hydrauliques qu'a nécessités l'équipement de cette centrale, en particulier le barrage qui, avec ses $61 \mathrm{~m}$. de hauteur, est l'ouvrage le plus haut de France, à ce jour encore, et crée un réservoir de $54.000 .000 \mathrm{de}^{3}$. Il étudie ensuite son armement mécanique et électricue.

La centrale, imposant bâtiment de $75 \mathrm{~m}$. de long, $15 \div 6 \mathrm{~m}$. de large) comporte 5 groupes en service et 1 en réserve, turbines de 150.000 CV., accouplées directement à des alternatives de $125.000 \mathrm{KVA}$. produisant du courant à $10.500 \mathrm{~V}$.

Les rendements obtenus atteignent $84 \%$ à pleine charge; $79 \%$ à $1 / 2$ charge - sous chute normale - et l'accélération due à une brusque suppression de la charge ne dépasse point $10 \%$ à vitesse normale.

Les services auxiliaires sont alimentés par 2 alternateurs de $650 \mathrm{KVA}$., sous 220 volts, ainsi que par une batterie d'accus, de 3.000 ampères $\mathrm{H}$.

La sous-station de transformation - d'un type tout nouveau - est installée "à ciel ouvert " et représente, dans l'ẻtude, ainsi que le schéma général des connections b. Enfin, sont encore examinées les lignes de transport de l'énergie, qui amènent à Paris la puissance produite à Eguzon, en attendant que soit terminée l'électrification de la partie du réseau $P, O$. qui la doit absorber dans l'avenir.

Génie Civil, 3 aoùt 1926.
J, B. 